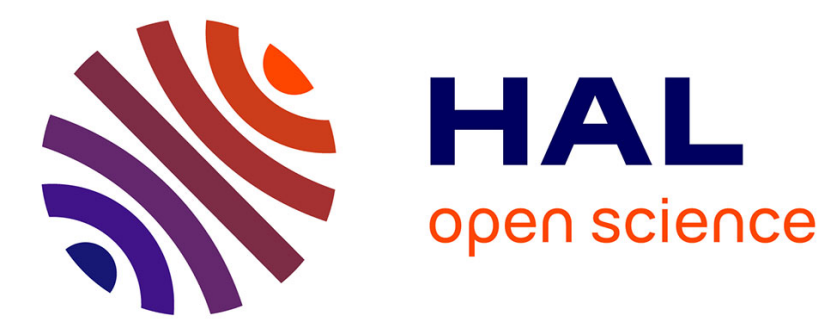

\title{
The Dynamics of Deictic Thoughts
}

Jérôme Dokic

\section{To cite this version:}

Jérôme Dokic. The Dynamics of Deictic Thoughts. Philosophical Studies, 1996, 2 (2), pp.179-204. ijn_00000139

\section{HAL Id: ijn_00000139 \\ https://hal.science/ijn_00000139}

Submitted on 5 Sep 2002

HAL is a multi-disciplinary open access archive for the deposit and dissemination of scientific research documents, whether they are published or not. The documents may come from teaching and research institutions in France or abroad, or from public or private research centers.
L'archive ouverte pluridisciplinaire HAL, est destinée au dépôt et à la diffusion de documents scientifiques de niveau recherche, publiés ou non, émanant des établissements d'enseignement et de recherche français ou étrangers, des laboratoires publics ou privés. 


\title{
THE DYNAMICS OF DEICTIC THOUGHTS*
}

\author{
Jérôme Dokic
}

0. This paper deals with the cognitive dynamics of deictic thoughts, i.e. thoughts expressed using indexical words like "this", "that", "this man", or "that man", and typically based on perception. The structure of the paper is the following. In $\S 1$, I try to circumscribe a typical problem raised by cognitive dynamics, which is to specify the conditions under which it is possible to change one's mind as time goes by. To explain what happens when a rational subject changes his mind, we need a prior account of the individuation of thought over time. In particular, we have to identify the deictic thought toward which a given change of attitude is directed. However, the well-known Fregean criterion can only be used to assess differences between thoughts held at the same time, and is powerless to compare thoughts held at different times. The criterion leaves open two contradictory alternatives for the individuation of deictic thoughts over time.

The first alternative is discussed in §2. It involves an extension of Gareth Evans' notion of a dynamic temporal thought, which is applied here to deictic thought. To express the same deictic thought at different times, it is necessary to exercise continually the capacity to keep track of the designated object. Continuity is constitutive of deictic thought itself. The second alternative is examined in $\S 3$. Continuity in keeping track is detached from the individuation of deictic thought. Strictly speaking, it is not thought which is dynamic, but particular cases of grasping a thought. It follows that deictic thought is not diachronically transparent: a subject can express the same deictic thought at different times without knowing it. Some reasons are given to prefer this alternative to the first one.

Assuming that the second alternative is correct as far as the individuation of deictic thought is concerned, I formulate in $\S 4$ two principles of an adequate theory of cognitive dynamics. A third principle is envisaged in $\S 5$, which concerns the issue of when a subject can be said to be internally justified in drawing a given conclusion from premisses held at (possibly) different times. At this point, a connection is made between the notion of dynamic grasp and that of justification.

In $\S 6$, the theory of deictic thought defended in the preceding sections is used in the context of a general argument according to which this kind of thought is de re, i.e. dependent on the particular object it is about. This argument is intended to illustrate the relevance of dynamic considerations for the definition of deictic thought. This suggests a further principle of cognitive dynamics, which relates the conditions under which it is possible to grasp continually the same thought to the semantic nature of that thought itself.

I conclude with a brief section (§7) in which I try to sketch a more encompassing definition of cognitive dynamics which does not concern only indexical expressions.

1. I borrow the phrase "cognitive dynamics" from David Kaplan, who formulates, without trying to solve it, what he probably sees as a central problem raised by cognitive dynamics:

Thus the problem of cognitive dynamics can be put like this: what does it mean to say of an individual who at one time sincerely asserted a sentence containing indexicals that at some later time he has (or has not) changed his mind with respect to his assertion? (1992: note 64, p. 538) 
Kaplan also says that a theory of cognitive dynamics deals with the question of what "standard adjustments" one must make in the formulation of one's indexical thought in order to express the same opinion as time passes. For example, Frege suggested (in "Der Gedanke") that the thought one can express on Monday by the words "Today is a beautiful day" can be expressed the day after by the words "Yesterday was a beautiful day". Accordingly, if I remark on Monday "Today is a beautiful day" and I say on Tuesday "Today is an awful day", I have not necessarily changed my mind about Monday. I can exploit the same linguistic form to express different thoughts. If I would like to express on Tuesday my original thought, I need to go from "today" to "yesterday", and from the present tense to the past tense. According to Kaplan, a description of these adjustments is precisely part of a theory of cognitive dynamics. However, this description may be less interesting in the case of deictic thought, since it seems to concern only the passage from "this" to "that" and vice-versa. Let us concentrate, then, on the more fundamental question of the conditions under which the subject who grasps a deictic thought changes (or does not change) his mind.

We can formulate a necessary and intuitive condition for a change of mind in general. When a rational subject changes his mind, he has first a given epistemic attitude toward a thought (for example, he believes that it is raining) and has later a contrary epistemic attitude toward the same thought (for example, he doubts that it is raining). For the time being, let us define the term "contrary" in the following way: no person can be right (i.e. justified) in holding simultaneously contrary attitudes toward the same thought. If I believe that $p$, and at the same time doubt that $p$, I am rationally committed to revise at least one of these attitudes. To be sure, this intuitive formulation of a change of mind raises many questions about the proper definition of rationality, and the possible fragmentation of the mind, but for now I am interested in a more immediate difficulty associated with it, which is specifically connected with cognitive dynamics.

Consider the following example. ${ }^{1}$ In a party, I am introduced to Igor, a brilliant chess player. At that time $\left(t_{1}\right)$, I think (truly) "This man is a great chess-player". But some time later, I remember that Igor has a twin brother, Boris, who is not particularly interested in chess. For some reason, I come to believe, of the person I met at $t_{1}$, that he was not Igor. I now think that he was Boris. I say to myself: "Sure, he looked like Igor, but in fact he was Boris, his twin brother". Unfortunately, I am wrong, because in fact I met Igor, and Boris was not even present at the party. Now as a result of my new belief about the identity of the person I met at $t_{1}$, I (unhappily) change my mind about his chess capabilities: I falsely think something like "After all, this man is not a great chess-player".

Now let us complicate the example a bit. Let us suppose that my change of mind occurs at some point between $t_{1}$ and $t_{2}$. At that latter time, I meet Igor at the same place again. I am persuaded that the man I meet at $t_{2} i s$ Igor - this time, someone trustworthy introduces him to me as Igor. (Note that I still have the false belief that the man I first met was Boris.) So I think "This man is a great chess-player". Both times $\left(t_{1}\right.$ and $\left.t_{2}\right)$, I used the same words to describe the same state of affairs: that Igor is a great chess player. However, as the example goes, there is a relevant change of mind in between.

In order to give a correct description of this change of mind, we have to identify the various firstorder thoughts I grasped. We cannot determine what counts as contrary attitudes to the same thought if we do not know how to individuate the thoughts expressed at $t_{1}$ and $t_{2}$. In fact, we can make a distinction 
between three potentially different thoughts. The first thought is expressed at $t_{1}$ by "This man is a great chess-player", and it is based on my perceiving Igor at that time. The second thought is expressed at $t_{2}$ by "This man is a great chess-player", and it is also based on my perceiving Igor at that time. The third thought is expressed at $t_{2}$ by "This man was not a great chess-player", and it is based on my remembering Igor at that time. Here the phrases "perceiving/remembering Igor" are understood in the so-called neutral or non-epistemic sense. In that sense, I can perceive Igor or remember him without perceiving or remembering that he is (was) Igor. ${ }^{2}$ We can extract from these thoughts three potentially different senses expressed by the deictic term "this man":

$\begin{array}{ll}\text { At } t_{1}: & \text { At } t_{2}: \\ \begin{array}{ll}\text { sense }_{1} \\ \begin{array}{l}\text { [based on the current } \\ \text { perception of Igor] }\end{array}\end{array} & \begin{array}{l}\text { sense }_{2} \\ \text { [based on the current } \\ \text { perception of Igor] }\end{array} \\ & \begin{array}{l}\text { sense } \\ \text { [based on the memory of Igor] }\end{array}\end{array}$

What are the relations between sense ${ }_{1}$, sense $_{2}$ and sense ${ }_{3}$ ? In a Fregean framework, a criterion of difference of thoughts similar to the following is generally accepted for the individuation of thoughts considered as the senses of complete sentences:

If, at a given time, we have two mental episodes of grasping a thought by a single subject, the thoughts grasped through these episodes are different if and only if the subject can rationally hold contrary attitudes toward them at that time.

To take a familiar example, if the subject associates, at a given time, different predicative pieces of information with the proper names "Hesperus" and "Phosphorus", he will be disposed, at that time, to accept a sentence of the form "Hesperus is F", where "F" expresses one of these pieces of information, but not a sentence of the form "Phosphorus is F", for the same F. According to the Fregean criterion (with the help of a suitable compositionality principle), this should entail that the proper names have different senses (at least for the subject).

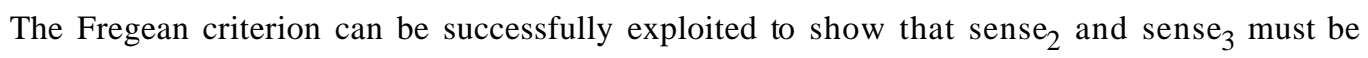
different, since I judge at the same time $\left(t_{2}\right)$ that this man [perception] is a great chess player, but that this man [memory] is not a great chess player. In fact, the interesting question concerns the relationship between sense ${ }_{1}$ and sense $_{2}$. Either sense ${ }_{1}$ and sense ${ }_{2}$ are different, or they are the same. Now the immediate difficulty lies in the application of the intuitive formulation of a change of mind. In fact, it seems that we have got into a circle. The Fregean criterion can be applied only to thoughts which are grasped simultaneously. To apply it over time, we need to suppose that the subject has not changed his mind between the relevant times, i.e. that his epistemic attitudes remained the same. (One's epistemic attitudes toward a given thought may change over time.) This supposition, though, can be made only if we already have a criterion of sense which enables us to compare thoughts held at different times.

This suggests a significant limitation of the technical notion of Fregean sense. ${ }^{3}$ According to the standard definition, Fregean sense is synchronically transparent, where the term "transparency" is 
introduced by Dummett in the following way: sense is (synchronically) transparent if and only if the subject who grasps simultaneously the senses of two synonymous signs cannot fail to realise that these signs have the same sense. ${ }^{4}$ Of course, the synchronic transparency of sense is disputable (cf. for example the problems connected with what is called the "paradox of analysis"), but the point I want to raise now is that even if sense is synchronically transparent, we have no reason to suppose that it is diachronically transparent. It is prima facie possible for a rational subject to grasp the same sense at different times without knowing it.

In other words, we have two alternative conceptions of the relations between sense 1 and sense . $_{\text {. }}$ The Fregean criterion cannot by itself settle the question of which conception is the right one. The criterion is necessarily synchronic, while sense ${ }_{1}$ and sense ${ }_{2}$ are expressed at different times. These conceptions result in incompatible descriptions of my change of mind. Suppose, on the one hand, that sense $_{1}$ and sense $_{2}$ are different senses. A natural proposal, at this point, is that I have grasped continuously the same sense, based first on perception, then on memory. We can say, then, that I have adopted contrary attitudes toward the thought expressed by "This man is a great chess-player": at $t_{1}$, this thought was based on perception and I endorsed it; at $t_{2}$, this thought was based on memory and I rejected it by endorsing the negation of this thought, expressed with the words "This man was not a great chess-player".

Suppose, on the other hand, that sense ${ }_{1}$ is the same as sense ${ }_{2}$. Ex hypothesi, I do not know that they are identical - sense is not diachronically transparent. To describe my change of mind in Igor's example, we cannot directly invoke the perception-based thought expressed on the first occasion by "That man is a great chess-player" and the memory-based thought expressed later by "That man is not a great chess-player", for the deictic senses involved are different in kind: since sense ${ }_{1}$ and sense $_{2}$ are the same sense, and sense ${ }_{2}$ and sense ${ }_{3}$ are different senses, we must conclude that sense ${ }_{1}$ is different from sense . $_{3}$. In fact, if we exclude borderline cases, there are two possibilities. Either I changed my mind on the first occasion, while still perceiving Igor, and I went from a perception-based thought to a contradictory one, or I changed my mind just after I perceptually lost track of Igor, and I went from a memory-based thought of the form "That man is a great chess-player" to the memory-based thought expressed later by "That man is not a great chess-player", where "that man" expresses the same sense each time. ${ }^{5}$

The alternatives distinguished here will be examined in the next two sections. As we shall see, the second alternative, according to which sense is sense $_{2}$, even though the subject does not know it, is superior to the first one, according to which these senses must be different.

2. Consider Evans' notion of a dynamic thought. Evans introduces this notion in the context of Frege's example involving the sentences "Today is fine" and "Yesterday was fine", which can express the same dynamic thought at two successive days $d_{1}$ and $d_{2}$. According to Evans, this is possible only if the subject who grasps this thought keeps track of the same day over time. As he writes:

[T] he way of thinking of an object to which the general Fregean conception of sense directs us is, in the case of a dynamic Fregean thought, a way of keeping track of an object. This permits us to say after all that a subject on $d_{2}$ is thinking of $d_{1}$ in the same way as on $d_{1}$, despite lower level differences, because the thought-episodes on the two days both depend upon the same exercise of a capacity to keep track of a time (1985:311). 
The lower level differences to which Evans alludes include the fact that at $d_{1}$ and $d_{2}$, the subject used different indexical words, i.e. "today" and "yesterday", to refer to the same day. Surely there are other differences, having to do for example with the subject's dispositions to act: I do not act in the same way when I think "The meeting is today" and "The meeting was yesterday". In the first case, I might get a move on, but in the second case, I might slow down. However, despite these differences, it is possible for the subject to express the same sense by using "today" and "yesterday" at different times. What counts here is the fact that he grasped that sense over time, grounded on a continuous exercise of a general capacity to keep track of an object. It is through this exercise that the object - a particular day - is presented in the same way to the subject.

We must be careful about the meaning we give to the phrase "grasping a sense over time". (Similar remarks apply to the phrase "grasping continuously the same sense" used here with the same meaning.) First, we can follow Dummett in making a distinction between the episodic and the dis positional reading of the phrase "grasping a sense". ${ }^{6}$ The episodic reading applies to cases where there is an act which actually expresses the relevant sense - for example, a supposition, a judgment or a speech act. The dispositional reading is supposed to deal with cases where there is no mental nor linguistic act which expresses the sense, although we still want to say that the subject grasps it. My disposition to use a sentence or to make a judgment involving a sense may be realised or not, but on the dispositional reading, I can still grasp the sense even if the disposition is not realised. Second, when we say that a subject grasps a temporal indexical sense over time, we mean that he has a permanent disposition to express that sense at particular times. Evans' idea is that to maintain this disposition in place, the subject has to exercise another capacity, which can be described as a capacity to keep track of something over time.

It is not a priori guaranteed that the sentence "Yesterday was fine", uttered at $d_{2}$, expresses the same thought as the sentence "Today is fine", uttered (by the same subject) at $d_{1}$. The subject may have lost track of time from $d_{1}$ to $d_{2}$, believing for example that two days have passed instead of one. In other words, the requirement of keeping track of time is not a trivial one, for it depends on the fallible ability to appreciate, even in a rough way, the passage of time, and to judge the thought as true or false according to how things are on the relevant day. If the subject loses track of this day from $d_{1}$ to $d_{2}$, he won't be able to grasp the same sense over that stretch of time. ${ }^{7}$

In Evans' conception, an utterance of "today" and an utterance of "yesterday" express the same sense only if they are connected by a single exercise of a general capacity to keep track of a particular day. If we extend this conception to deictic thought, we get close to the first alternative distinguished in $\S 1$. More precisely, we have the suggestion that two utterances of "this man" express the same sense only if they are connected by a single occurrence of the capacity to keep track of a particular man. In the Igor example, sense ${ }_{1}$ and sense $_{2}$, i.e. the perception-based senses expressed at $t_{1}$ and at $t_{2}$, are surely not connected by an exercise of the ability to keep track of Igor from $t_{1}$ to $t_{2}$. I do not even believe that the man I saw at $t_{1}$ is the same as the one I saw at $t_{2}$. Consequently, sense ${ }_{1}$ is not the same sense as sense ${ }_{2}$.

However, it is open to the friend of Evans' conception, as it is extended to deictic thought, to claim that in a refined sense of the phrase "keeping track", sense $_{1}$ and sense $e_{3}$, i.e. the perception-based sense expressed at $t_{1}$ and the memory-based sense expressed at $t_{2}$, are connected by an exercise of keeping track of Igor. First, I keep track of Igor by perceiving him, and then I continue to keep track of him 
by remembering him. It would follow that sense is the same sense as sense $_{3}$. This claim will be discussed further in the next section, but if it is made, we can sum up the first alternative as follows. First, sense $_{1}$ is different from sense $e_{2}$, because in one way, the subject has lost track of Igor from $t_{1}$ to $t_{2}$. My two acts of perceiving Igor are not connected by an exercise of the capacity to keep track of him from $t_{1}$ to $t_{2}$. Second, sense $_{1}$ is sense $_{3}$, since in another way, the subject has kept track of Igor. More precisely, he grasped the same sense from $t_{1}$ to $t_{2}$, based first on perception, then on memory.

3. In the case of temporal indexical thought, the thesis that identity of sense depends on a single exercise of the capacity to keep track of time may seem mandatory. Indeed, on one way of looking at the matter, if one loses the indexical track of time, one cannot put oneself on the same track again. Suppose for example that on Monday, I think "Today is fine". On the morning of the next day, I come to believe (wrongly) that I have been drugged and have slept for one week. Since I have lost track of time, I cannot grasp on Tuesday the sense expressed the day before by the word "today". My use then of the word "yesterday" would not be based on a proper appreciation of the passage of time. Moreover, I cannot grasp that sense again, for I have definitely lost it. I cannot travel in time, so I am unable to go back to Monday in order to think of this day as "today" again.

Is there not another way of looking at the matter, according to which it is possible for me to grasp the same temporal indexical sense again? Later in the day, for instance, I could realise that I have not been drugged, and that I have slept one night as usual. Can't I grasp again the sense expressed by "today" on Monday? Maybe, but if we claim that I can grasp that sense again, we have to concede that there was a (non-fatal) interruption in a single exercise of the capacity to keep track of time, and not that I begin a new exercise of that capacity. On either way of looking at the matter, there do not seem to be two alternatives about the individuation of temporal indexical sense, between which one has to decide, as there are two alternatives about the individuation of deictic sense. The relevant question is whether distinct exercises of the capacity to keep track of an object may ground the same deictic thought about it. According to the first alternative, the answer is negative; it is positive according to the second one. Since Evans concentrated mainly on the cognitive dynamics of temporal indexical thought, it is no surprise that he did not explicitly address this question.

In the case of deictic sense, it is prima facie possible to grasp the same sense again without knowing it, because one can meet the same object at different times without realising that it is the same one again, and thus initiate distinct exercises of the capacity to keep track of it. Let us then consider this possibility with respect to the Igor example. The suggestion is that $\operatorname{sense}_{1}$ is the same as sense ${ }_{2}$, even if the utterances of "this man" based on perceptual acts at $t_{1}$ and $t_{2}$ are not connected by an exercise of the ability to keep track of Igor. Indeed, the issue of the identity and difference of these senses is detached from the question of whether the subject has continuously grasped the same sense from $t_{1}$ to $t_{2}$. More generally, continuity is no more constitutive of deictic thought, even though it is required for grasping such a thought over time.

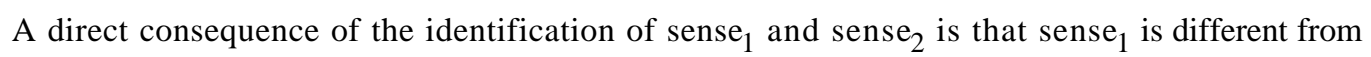
sense $_{3}$, since the Fregean criterion predicts anyway that sense ${ }_{2}$ is different from sense $e_{3}$. Which alternative is the best one? Of course, the difference in kind between a perception-based sense (e.g., sense ${ }_{1}$ ) and a 
memory-based sense (e.g., sense ${ }_{3}$ ) has yet to be confirmed by a substantial theory of thought. In fact, Evans himself notes elsewhere that one is naturally, but not automatically placed in a position to make a judgement based on the memory of an object which was just perceived. In fact, according to Evans' theory, quite different mechanisms of (non-descriptive) identification are in play depending on whether a memory-based or a perception-based sense is grasped. ${ }^{8}$ However, I think that a consideration independent from the details of a theory of thought favours the thesis that sense ${ }_{1}$ is sense $_{2}$.

The point is that the first alternative results in a multiplication of kinds of perception-based senses (and deictic senses in general) beyond necessity. Suppose, to take another example, that the subject did not change his mind, because he knew all along that Boris was not at the party - that the only man he saw was Igor. He expresses his thought in the same way at $t_{1}$ and $t_{2}$ : "This man is a great chessplayer". Can we say that in this new case, the subject has retained a single deictic sense from $t_{1}$ to $t_{2}$ ? The answer to this question depends on what we think happened in between. An obvious suggestion is that when he does not currently perceive Igor, the subject grasps a memory-based sense of him. So we have a single deictic sense, first based on perception, then on memory, then on perception again.

This suggestion, though, does not work. Here is why. In this example as in the original one, we suppose that a man is perceived, then unperceived for some time. During that time, the subject grasps a deictic sense of him which is based on memory. Now it seems that were the same man to be perceived again, the subject could always have doubts as to whether the man remembered is the same as the man currently perceived. Of course, these doubts may not be reasonable in the light of the subject's collateral attitudes (for example, his knowledge that Boris has not been invited), but it does not mean that they are not rational by themselves. The modal dimension of the Fregean criterion invites us precisely to abstract from such collateral attitudes, and to consider whether the rational subject can have these doubts, even if he actually does not doubt. (In a Fregean framework, the fact that a subject does not actually doubt that Hesperus is Phosphorus does not ipso facto imply that "Hesperus" and "Phosphorus" have the same sense.) It seems that the subject can conceive of a substitution of the man first perceived, at least if the time during which he perceptually lost track of him was long enough. A consequence of the first alternative seems to be this: as long as the subject still grasps a memory-based sense which was once based on perception, it is not possible for him to grasp this sense again through perception. In other words, once we allow a perception-based sense to change into a memory-based one, because the object left the perceptual field, we cannot allow the latter sense to return to its original perceptual form.

It may be objected that I underestimate the differences between our original example and the case just discussed, in which the subject knows that Boris is not at the party. In the latter case, my memory of Igor is not the only aspect which contributes to bridge the epistemic gap between my two acts of perceiving him: my whole thought and reasoning operates under the supposition that I know where Igor is even when I do not perceive him, because no one else at the party looks exactly like him. This supposition cannot be considered merely as a collateral piece of information, given the substantial role it plays in my thought and reasoning: I exercise continuously a (not wholly conceptual) capacity to keep track of Igor, not only through my current perception of him, but also when he is unperceived, through my knowledge of where he is roughly, relative to me at each time. Then some suppositions, and some capacities, which are absent in the original example, are constitutive of the perception-based sense grasped in the second 
case. Of course, the relevant question concerns the conditions under which it is possible to keep track, in this extended sense, of an object over time. According to John Campbell, who defends this account of deictic sense, these conditions include my ability to trade directly upon the fact that my two uses of "that man" are co-referential. When sameness of reference is manifest, i.e. when it is obvious to me that I meet Igor again at $t_{2}$, I can grasp the same deictic sense from $t_{1}$ to $t_{2}{ }^{9}$

There are two reasons to think that this account is not very economic as far as the individuation of deictic sense is concerned. First, it seems that the deictic sense involved in the example just discussed has to be distinguished from the deictic senses involved in the original example. The first sense may be based indifferently on perception or on memory, while each of the latter senses has to be based either on perception or on memory, but not on both. Indeed, Campbell admits that the first sense is in some way "compound" (presumably because different faculties and attitudes are involved in grasping it), but insists that it may not be decomposed in simpler senses: "At the foundations of our talk of objects, there are not such simple senses to be had." 10

I agree that there actually may be few perception-based senses which are simple, i.e. purely based on perception: I often recognise familiar objects through perception, and grasp a compound sense, based partly on memory, and partly on perception. The notion of a compound sense, though, is still the notion of a composition of simple senses, and the subject can always stick to a simple deictic sense if he brackets out the fact that he recognises the object as (numerically) the same again. In that case, the subject simply perceives an object, and this is enough (no doubt in the context of properly conceptual capacities) for him to grasp a simple deictic sense.

Even if we set aside, though, the issue of the nature of simple senses, there is a second reason why Campbell's account multiplies deictic senses without necessity. His account implies that if the subject grasps a deictic sense of an object, and then loses track of it (in the extended sense suggested by Campbell), he may not be able to grasp the same deictic sense again. Campbell gives the example of a subject who makes the judgements "That wasp is F" and "That wasp is G" while he is keeping track of the same wasp fromone judgement to the other. In that case, the thoughts involved in these judgments have a deictic sense in common (expressed by "that") but, Campbell adds, "if the subject had not succeeded in keeping track of the wasp, then he would have been unable to grasp either of those thoughts". ${ }^{11}$ Indeed, in that case, the identity of reference is not manifest to the subject.

Contrast the second alternative. A perception-based sense can be grasped again, at least by the same subject, and the question of whether he has or has not memories of earlier perceptual encounters is irrelevant. It is still true that when the subject grasps the same deictic sense over time, he can "trade upon" the fact that his two uses of "that" are co-referential, but it does not imply that when the subject is unable to trade upon this fact, these uses cannot express the same sense. My hypothesis is that they may express the same sense, even though this is not obvious even to the rational subject. ${ }^{12}$ Obviously, I make some kind of mistake in believing that I have not been presented both times with the same man. However, I may be excused for my mistake since it does not impair my rationality: to compare sense ${ }_{1}$ and sense ${ }_{2}$ in the absence of any piece of collateral information, I would have to take a point of view outside my temporal experience which would be at the same time a deictic point of view, and this I cannot do. (If a god could 
adopt such a point of view, he would perhaps realise that those senses are identical, even without any piece of collateral information.)

Let us sum up the second alternative. When I first saw Igor, I grasped a deictic sense of him which was based on perception. I kept track of this sense because I perceptually kept track of the same man. When Igor left my perceptual field, though, I lost track of this sense. Of course, I retained pieces of information from the original encounter, which I could express by using a sentence like "That man was F", where the sense expressed by "that man" is based on the memory of the man I perceived at the first time (Igor). When I saw Igor for the second time, I grasped the same perception-based sense, but I did not know it, because I was unable to compare this sense with the one grasped at the first encounter, which was subsequently lost.

According to the second alternative, a perception-based sense is essentially based on perception. We cannot grasp such a sense if we do not currently perceive the designated object. When the object leaves the perceptual field, we lose track of this perception-based sense, and we naturally come to grasp another sense, based on the memory of the object which was just perceived.

4. I have argued that among the alternatives distinguished in $\S 1$, the second is theoretically better than the first. In this and the next two sections, I try to extract from our discussion so far four principles of cognitive dynamics. The first may be called the principle of continuity:

CD1. To explain how a subject grasps the same sense over time, it is not enough to point out that the subject grasps the same sense at every time within the relevant period.

In the Igor example, the mere fact that the subject grasps the same perception-based sense at different times does not guarantee that he grasps this sense in a continuous way from one time to another. Of course, the following fact may always be true: that when it is correct to say that a subject grasps the same sense at every time within a given period, it is also correct to say that he grasps that sense in a continuous way during this period. However, this does not falsify the principle of continuity, which is about explanation: a theory of cognitive dynamics has to deal with internal continuity, i.e. with the diachronic relations between different times at which the thought is grasped, and not merely with synchronic data relative to the thought as it is grasped at given times. Once we admit that sense is not always diachronically transparent, the definition of these relations becomes a central task for such a theory.

Let us say that when a subject grasps a sense which determines an object in the world, he is intentionally related to that object. Now according to the principle of continuity, the notion of internal continuity requires more than the mere permanence of a given intentional relation. What else does it require? A plausible suggestion is contained in the following principle, which we may call the principle of activity:

CD2. A subject grasps the sense of a sign over time only if he exercises continuously his capacity to maintain the same intentional relation to the referent of the sign. 
According to this principle, there is an active dimension in forming a concept, or grasping a sense. Grasping the sense of a sign does not only require a definite capacity, it is also tied to an activity (of course not in the sense of a conscious striving). The subject who grasps such a sense does not master a concept once and for all but, metaphorically speaking, must make adjustments as time passes in order to keep himself in the same intentional relation to the world. Of course, a non-metaphorical characterisation of this activity of adjustment has to be given when specific kinds of senses are considered. ${ }^{13}$

The interpretation of the grasping relation as involving an epistemic activity contributes to explain further what one means by the notion of internal continuity. This kind of continuity is internal because it is up to the subject to maintain the continuity - continuity is not something that merely happens to one. Consequently, one may try to maintain the continuity without succeeding. We can contrast this notion of continuity with a different, less demanding notion. Consider an example adapted from M. Pendlebury. ${ }^{14}$ I am driving a visiting friend around the Geneva country-side. During the whole drive, I am disposed to accept the sentence "This is the canton of Geneva". However, I do not know that I frequently cross the boundary of the canton of Geneva, so that the reference of the indexical "this" is not stable: sometimes it designates the canton of Vaud, and the sentence I am disposed to accept is false; sometimes it designates the canton of Geneva, and the sentence I am disposed to accept is true. Intuitively, I have not changed my mind; in particular, I have perceived no new evidence which would have caused me to adjust the corpus of my beliefs.

If we take this example at face value, internal continuity does not imply that the subject keeps on thinking the same thing - at least if by "thinking the same thing", we mean being related to the same Fregean thought. The sentence "This is the canton of Geneva" would express different thoughts at different times. Does it mean that the notion of internal continuity must be detached from that of being related to the same thought? I do not think so. In the example just given, the subject does not keep track of the same referent over time, because he is not aware of each point of the boundary of the canton of Geneva, and this awareness would be relevant in that case. Maybe the subject's ignorance is harmless when he is right in the town of Geneva, but it may have unfortunate consequences on his thought and reasoning when he is in the country-side, where the risk of crossing the boundary of the canton of Geneva without noticing it is quite high. Whatever my public utterances would mean, then, there is no relevant internal continuity in my thought. All we can say is that it is as if there was internal continuity, without there being really such a continuity. ${ }^{15}$

Let us see more precisely how the principles just formulated apply to deictic thought. When the subject grasps a deictic thought at a given time, he is, at that time, intentionally related to an object mainly by perceiving it (again, only in the context of properly conceptual attitudes). Now according to the principle of activity, the subject who grasps that thought over time must exercise a capacity to maintain the perceptual contact with the object. We can give a partial, but non-metaphorical characterisation of this capacity: its exercise includes compensatory movements which correct specific transformations of the perceived reality. More precisely, we have to distinguish between three kinds of transformations. Transformations of the first kind do not affect the perceptual relation to the object; to this kind belong transformations of the perceptual background and many qualitative changes of the object. Transformations of the second kind may not affect the perceptual relation to the object, but only if the 
subject makes appropriate compensations for keeping track of the object. To this kind belong sensible spatial movements of the subject and/or the object, compensated by the subject's own movements (of his eyes, his head, or his whole body). Transformations of the third kind are fatal to the perceptual relation, as when the object definitely leaves the perceptual field. In this case, the subject loses track of the object, and with it the deictic sense.

What about the cases in which the perceptual scene is static, i.e. when no positive compensation is needed? In those cases, the subject is perceptually sensitive to possible transformations which belong to the second kind, in the following sense: if one of these transformations took place, the subject would make the appropriate compensation. The systematic description of this sensitivity, and of the way it is realised in the subject, is a task for a theory of perception. What is important here is that we can still claim that the subject exercises a capacity to maintain the perceptual contact with the object even if the spatial relations between the subject and the object do not actually change.

The notions of transformation and of compensation also contribute to the satisfaction of the principle of continuity. The capacity to keep track of a perceived object is manifested when a transformation of the perceived reality - e.g. the object's movement relative to the subject - is accompanied by a suitable compensation - the subject's active and readjusting movement. This manifestation is necessarily extended in time, since it involves at least two continuous moments. The case in which no positive compensation is needed is one of equilibrium: here, we cannot ascribe a deictic sense to the subject unless we refer to possible transformations of the perceived reality, and to his disposition to make corresponding compensations. The relations between transformations and compensations are precisely (among) the diachronic relations referred to in the principle of continuity.

One consequence of distinguishing sharply between the level of sense and the level of grasping the sense is that we can now consider deictic sense, in conformity with the second alternative formulated above, to be invariant under specific transformations of the perceived reality (in fact, non-fatal transformations of the first two kinds). The individuation of deictic sense can be abstracted from irrelevant properties of its grasping by a particular subject. Once again, the exercise of the capacity to keep track of something is not itself a constituent of the deictic sense.

5. We said that when the subject grasps the same sense over time, he can trade upon the fact that different expressions having that sense, as they are used within the relevant period, have the same referent. What we meant is that the subject is justified in doing so. There is an epistemological contrast between the case in which the subject keeps track of the same object over time, and the case in which he loses track of that object. In the last case, even if the subject grasps the same sense again, he may not be justified in trading upon the fact that different expressions having that sense, as they are used within the relevant period, are co-referential.

The same contrast may be formulated in terms of the arguments which the subject would be right to make. For example, if I perceptually keep track of an object over time, so that I can collect different pieces of information about it, I am justified in concluding, from a set of deictic thoughts of the form "This is F", "This is G", "This is H", etc., an existential thought of the form "There is something which is F, G, H, etc.". On the other hand, if I lose track of the object at some point between the times when I grasp the 
thoughts "This is F" and "This is G", I may not be justified in drawing the same conclusion, even though the deictic "this" expresses the same sense on both occasions. This suggests the following principle of justification:

CD3. The conditions under which it is possible to grasp the same sense over time should determine conditions under which it is right to draw an appropriate conclusion from a set of premisses involving that sense.

It is important to realise that the contrast just given is different from the one described by Lewis Carroll in his famous paper "What the Tortoise said to Achilles" (1895). In Carroll's story, the Tortoise (considered as a non-human person) refuses to draw a given conclusion which nevertheless follows logically from premisses accepted by him. This example shows inter alia that an argument, considered as an inferential passage from a set of premisses to a conclusion, is more like an action than something that merely happens to one. (At one point of Carroll's dialogue, Achilles says that Logic would take the Tortoise by the throat, and force him to accept a given proposition. Of course, this threat does not much impress the Tortoise.) Cognitive dynamics, though, raises another kind of problem, which concentrates on the difference between a person who would be right to draw a given conclusion from a set of premisses and a person who would not be right (at least not right in the same way as the first person) to draw the same conclusion from the same set of premisses. Most of the time, the first person, contrary to the second, actually draws the conclusion. However, it might be that while he is in the same epistemological context, the first person refuses to draw the conclusion (without rejecting it) - the Tortoise is such a person -, while the second judges the conclusion. If this happens, neither person is reasonable, and the second may be frankly irrational (as he may make a wild shot in the dark). ${ }^{16}$

Of course, the reason which the subject has for drawing the conclusion in the favourable case is not a propositional reason which comes in the form of a new premiss: rather, the justification comes in the way the subject grasps the premisses of the argument, and is not due to the dubious presence of another, silent premiss. Note that this way of describing the example is not necessarily incompatible with the (Davidsonian) thesis that justification relates only propositional thoughts: in fact, we still have to say something about the conditions under which this relation is recognised, or how it can be exemplified in particular grasping relations. Then, being right is always having a propositional justification. In the example, a subject can be right about drawing a certain conclusion, without having a propositional justification for drawing such a conclu sion other than his grasp of the original set of premisses.

6. I borrow the fourth and last principle from Peacocke, who calls it the principle of dependence. Peacocke does not introduce it in relation to cognitive dynamics, but its application to the dynamic case is worth exploring. Here is his own formulation:

CD4. There can be nothing more to the nature of a concept than is determined by a correct account of the capacity of the thinker who has mastered the concept to have propositional attitudes to contents containing that concept (a correct account of "grasping the concept"). ${ }^{17}$ 
The principle of dependence entails that once you have a correct account of grasping a Fregean sense, you also have a semantic account of that sense. I have argued for the thesis that continuity is not constitutive of deictic sense. In what follows, I give the sketch of an argument, which takes this thesis as one of its premisses, in favour of a substantial semantic interpretation of deictic sense. The semantic interpretation I am about to argue for is a familiar one: the perception-based sense expressed by deictic words is de re, i.e. dependent on the numerical identity of the designated object. ${ }^{18}$ This leads to the following proliferation of perception-based senses: at least one different kind of perception-based sense is associated with each possible object of ostension. However, we can try in many cases to motivate the de re character of such senses by considering the dynamic conditions under which we grasp them. Hence, the cognitive dynamics of deictic sense is directly relevant to the definition of that sense itself.

Here is the argument. Consider two objects $a$ and $b$, simultaneously presented in the perceptual field of a rational subject. The subject is free to hold (in fact, should hold) different epistemic attitudes towards these objects. Hence, the (synchronic) Fregean criterion of difference predicts that $t w o$ deictic senses are in play here (even if the objects look exactly the same). Suppose now that the subject fixes his gaze on $a$, without losing track of $b$. As it has been suggested, the deictic sense which determines $a$ is invariant under relevant transformations of the perceptual scene, including the fact that $b$ leaves the subject's perceptual field. What is relevant is that the subject should not lose track of $a$. Suppose then that a third object $c$ appears in the place of $b$. The same Fregean criterion predicts that the deictic sense associated with $c$ is different from the one associated with $a$, which is still the object of the subject's attention. By induction, we can show that the perception-based sense associated with $a$ is always different from the sense associated with any other object which can be simultaneously perceived. More formally, where "s $(x)$ " is a function whose value is a deictic sense determining $x$, we have:

$$
\forall x[x \neq a \rightarrow \mathrm{s}(x) \neq \mathrm{s}(a)]
$$

Obviously, this procedure does not show that the senses associated with $b, c, d$, etc., are different from each other. In other words, we have not proved the following yet, which formally defines the de re character of deictic sense:

$$
\forall x \forall y[x \neq y \rightarrow \mathrm{s}(x) \neq \mathrm{s}(y)]
$$

However, to prove this, a similar (and progressively shorter) procedure can be conducted for each of the other objects, considered in their turn as objects of persisting attention. The thesis that deictic sense can be grasped again at different times indicates that the earlier procedures fix an important property of the sense associated with the considered object (in our example, a), a property which can be referred to in the later procedures.

According to the foregoing argument, the de re character of deictic thought originates in the possibility of being simultaneously confronted, in perception, with more than one object. Of course, this argument is too schematic as it stands, and its use of induction has to be carefully examined. Besides, it does not seem to apply directly to every particular case (consider any big object which occupies the whole 
of the perceptual field, and cannot be perceived simultaneously with another one), but I think it is enough to illustrate a possible way in which dynamic considerations help to determine an important semantic property of deictic sense.

7. As they are formulated, the four principles of cognitive dynamics are not specific to deictic thought, though we cannot be sure in advance that they will apply in an interesting way to other kinds of thoughts. In particular, the principles of continuity and of activity have to be given a substantial interpretation if they are to deal with non-indexical thoughts. In this last section, I should like to conclude with some brief remarks on a programmatic theory of cognitive dynamics which would be valid even outside the sphere of indexicality.

In $\S 1$, we noted that a theory of cognitive dynamics should include an account of the conditions under which a change of mind is possible. A necessary condition of a change of mind, considered as a conscious exercise of spontaneity, is that the subject is able to grasp the same sense over time. If this condition is not satisfied, we cannot say that the subject has intentionally modified his epistemic relation to a given thought. The satisfaction of this condition fits an "internalist" requirement according to which, when a subject changes his mind at a certain time, he must be at least disposed to know, at that time, that he is changing his mind. ${ }^{19}$ Now we have envisaged a theory of cognitive dynamics which is not concerned with changes of mind as such, but rather with the conditions of internal continuity, i.e. the conditions under which the grasping of a sense persists over time. Once we have an account of cognitive dynamics in this sense, we can invoke in a non-circular way the intuitive formulation of a change of mind given in the first section.

We need to distinguish carefully two questions here. In the first place, there is the question as to whether it is possible to grasp the same sense at different times. In the second place, and only if the answer to the first question is affirmative, there is the question of how we can grasp this sense in a diachronic or continuous way. We have supposed here that there is an affirmative answer to the first question but obviously, the fate of the theory of cognitive dynamics depends on there being a convincing justification for this supposition. However, it is enough to concentrate on the second question, since as soon as we have a plausible model of cognitive dynamics, we also have the beginning of an answer to the first question.

As internal continuity is prevalent in human intellectual activities, a complete theory of cognitive dynamics would have to explain no less than the possibility of science and reasoning. We can collect different pieces of information about the same object and thus elaborate theories about it only if we have the means of grasping the same sense of that object as long as our investigation lasts. Complex inferences have normally at least one middle term, and thus exploits the same sense in different premisses held at different times. ${ }^{20}$ To illustrate the problem in a dramatic way, let us consider the following inferential arguments:

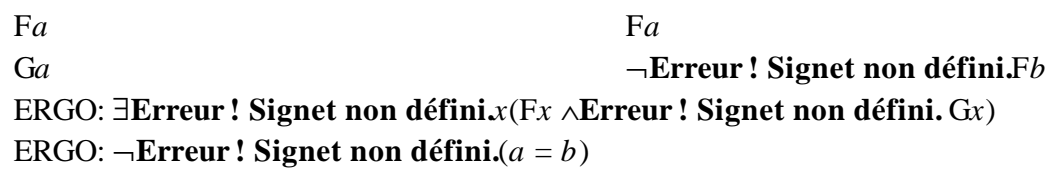


We have to suppose that these arguments are concrete inferences made by a particular subject: each premiss is constituted by a distinct occurrence of a complex sign used by the subject. It follows that these inferences are not exactly implications in the sense relevant to formal logic. In general, we can make a distinction between deductive implications, which involve complex logical relations between thoughts, and deductive inferences, which are mental actions leading the subject from a set of premisses to a conclusion. $^{21}$ Deductive implications are atemporal and the fact that they are valid does not typically depend on a particular reasoning subject. Deductive inferences, on the other hand, have to be ascribed to a particular subject, and are normally extended in time. The precise relationship between a theory of deductive implications and a theory of deductive inferences is still unsettled, as one can judge from the recent literature on the issue of psychologism in logic. I think that an adequate theory should have something to say on that complex issue, even though it concerns mainly the level of deductive inferences, and not the level of deductive implications.

From a logical point of view, the conditions of validity of these arguments are reasonably clear: they include at least the fact that the occurrences of the constant " $a$ " designate the same object, and the fact that the occurrences of the predicate letter "F" express the same property. However, given the logical validity of these arguments, we have to ask what the conditions are under which the subject is placed in a position to recognise that they are valid. We have to explain the possible difference between an "unfavourable" case in which the subject understands all the premisses, but does not see any reason to draw the conclusion, and a "favourable" case in which the understanding of the premisses provides the subject with a good, internal reason to draw the conclusion. The permanent possibility of a gap between understanding the premisses and being right to accept the conclusion is due here to the fact that concrete reasoning necessarily takes (some) time..$^{22}$ This is surely the fundamental axiom of cognitive dynamics, on which the other dynamic principles are grounded. 


\section{NOTES}

* I would like to thank Curzio Chiesa, Bill Child, Eros Corazza, Fred Mermoud, Ben Morison, François Récanati, David Wiggins and two anonymous referees for helpful comments on earlier versions of this paper.

${ }^{1}$ Similar examples are discussed by Branquinho (1992). However, my preferred theoretical description of the current example, in $\S 3$, differs from Branquinho's in a crucial way.

${ }^{2}$ For this distinction, cf. Dretske (1969).

${ }^{3}$ This limitation is also noted by Evans (1985: 308).

${ }^{4}$ Cf. Dummett (1973: 95).

5 The second alternative implies that no change of mind can ever be a direct relation between the perception-based thought "This man is a great chess-player" (expressed at $t_{1}$ ) and the memory-based thought "This man is not a great chess-player" (expressed at some point between $\mathrm{t}_{1}$ and $\mathrm{t}_{2}$ ). Is this counter-intuitive? I do not think so. On either alternative, it is plausible to require that the subject must have some grasp of the thought in respect of which he changes his mind. One way of dealing with this requirement is as follows. A change of mind may be seen as an epistemic response to a situation in which the subject has contradictory beliefs, and knows that they are contradictory. This response involves at least a contraction: the older belief is removed from my doxastic system (cf. Forrest, 1986). So when I change my mind between $t_{1}$ and $t_{2}$, I remove from my doxastic system the thought "This man is a great chess-player". This leaves completely open the question of whether this latter thought is the same as the one expressed at $\mathrm{t}_{1}$.

${ }^{6}$ Cf. Dummett (1988: ch. 9).

${ }^{7}$ The question of what thought, if any, is expressed at $d_{2}$ can be put aside here. The fallibility of the keeping track capacity is also stressed by John Campbell in his important paper (1987).

${ }^{8}$ Cf. (1982: 267). Evans' claim implies that sense ${ }_{1}$ cannot be the same as sense $e_{3}$. If we combine this claim with the thesis that sense ${ }_{1}$ is not the same as sense $_{2}$ (also made by the friend of the first alternative), we get another alternative yet, distinct from the ones discussed here. However, the arguments which will be levelled against the first alternative can also be used against this one.

${ }^{9}$ Cf. Campbell (1987), followed here by Branquinho (1992).

${ }^{10}$ Campbell (1987: 280).

${ }^{11}$ Campbell (1987: 285).

${ }^{12}$ Indeed, we can question Campbell's views on the individuation of deictic sense without relying on what he calls a "Cartesian" conception of inference, according to which the capacity to grasp the same sense over time is infallible.

${ }^{13}$ Perry recognises the need of introducing an active dimension to deal with cognitive dynamics. He suggests that there is a sense in which one can try to preserve the proposition believed (a Russellian proposition, in his preferred framework): cf. "A Problem about Continued Belief", reprinted in his 1993. However, it seems to me that he forgets this insight when he introduces later in the paper his own causal account: causality as such is a "passive" notion. The subject cannot be made responsible for the mere existence (or non-existence) of a causal relation.

${ }^{14}$ Cf. Perry (1993: 77-8).

${ }^{15}$ As before in the case of temporal indexical thought, I leave open the question of whether, in this example, the subject is related to different thoughts at different times, or to no coherent thought at all.

${ }^{16}$ We could say that in the favourable case, the Tortoise has a disposition to draw justifiably the relevant conclusion but, for some reason (perhaps because he is stubborn), does not actualize that disposition.

${ }^{17}$ Cf. Peacocke (1992: 5). As Peacocke says, the principle of dependence is the concept-theoretic analogue of a principle about language formulated by Dummett in several of his writings.

${ }^{18}$ Cf. Evans (1982) and McDowell (1984).

${ }^{19}$ Of course, there may be more "external" notions of a change of mind, which allow that a subject may change his mind without knowing it, but according to the internalist requirement, these notions must be somehow parasitic on the internal conception of a change of mind. For example, in the canton of Geneva example described above, it may be said that there is a sense in which the subject changes his mind when he unknowingly crosses the boundary of the canton of Geneva. 
${ }^{20}$ This theme is discussed by Millikan; cf. what she calls the "act of identification" in her 1984 book. In Millikan (1991), she acknowledges the problems posed for the notion of Fregean sense by cognitive dynamics. However, a claim of the present paper is that such problems are not necessarily insuperable.

${ }^{21}$ Haack (1982) introduces this distinction.

${ }^{22}$ I take it that the intervention of a third premiss, which would either use or mention the constant " $a$ ", does not even begin to solve the problem. For a detailed discussion of this point, see the paper quoted in Perry (1993). Moreover, as Millikan (1991) has argued, it is not obvious that the internal representation of an identity between the references of two occurrences of singular terms requires a formal identity, let alone a formal continuity, between the relevant occurrences. 